\title{
Gamma Calibration and Phase Error Compensation for Phase Shifting Profilometry
}

\begin{abstract}
Wang Yang ${ }^{*}$, Feng Yao, Fan Jianying and Fu Yao
The Higher Educational Key Laboratory for Measuring and Control Technology and Instrumentations of Heilongjiang Province, Harbin University of Science and

Technology, Harbin, China

*Corresponding author: ceaser@126.com

\section{Abstract}

Phase Shifting Profilometry is a method of structired light those three-dimensional reconstructions are susceptible to error from Gamma distortion. In practice, the luminance nonlinearity caused by the Gamma distortion effect of d digitel video projector and a digital camera yields undesired fringe intensity changes, which substantially reduce the measurement accuracy, and while the effects of the Garing distortion diminish with an increasing number of employed phase-shifting patterns.y Gamma calibration method expressed as Fourier series and binomal series Cheorem is proposed to reduce the measurement error caused by the Gamma distortion. After Gamma calibration, the Gamma distortion effect is attenuated and the accuracy is enhanced. The linear least square is compensated the phase error of absolute ${ }^{\bullet}$ hase still existing after Gamma calibration. To verify the presented method in this paperca profilometry experimental system is constructed using digital video projector and CCD camera. The experiment results shown that, the Gamma calibration reduce the maximum phase error of absolute phase by $75.0 \%$. After the linear least square compensation, the maximum phase error is only $5.9 \%$ of without any Gamma calibration or phase compensation. The reconstruction surface of a complex curve surface is hardy any waviness, which is clearly noted in the reconstruction of the same tested object without any correction ror compensation.
\end{abstract}

Keywords: Fringe Projection Profilometry, Phase-shifting, Gamma distortion, Gamma Calibration, Phase Error Compensation

\section{Introduction}

Phase Shifting Profilometry (PSP) is a well-known optical metrology based on active vision. has been developed to meet the demands of various applications [1-2]. PSP is acquired the distortion fringe image by projecting structured-light patterns onto the objects, and by modulating the object surface of interest [3-4]. Because of the patterns and image as the fringe, PSP named as Fringe Projection Profilometry (FPP). Compared to other optical measurement methods, FPP, based on digital patterns-projection, unwrapped phase computation, and phase-shifting profilometry, and has the advantage of high resolution, high measurement speed; less sensitive to the surface reflectivity variations and the object can be measured point by point [5-6].

However, the measurement accuracy of FPP is usually affected by many error sources such as the phase-shift error, camera noise and nonlinearity, vibration, and speckle noise [7]. By used in FPP, the commercial Digital Video Projector (DVP) and the CCD camera are generally fabricated to be nonlinear devices for a better visual effect [8], and the Gamma 
distortion of DVP and CCD camera pairs is the most important factor for the measurement accuracy of 3D shape measurement. For the commonly used PSP, the phase errors caused by Gamma distortion are regarded as the dominant error source.

In order to reduce the phase error for gamma distortion, the first method uses a low-pass filter. This type of method was originally proposed by Carocci et al. for measurement systems based on the digital fringe projection [9]. Defocusing [8] method is belonging to this type. The second method is phase error compensation by interpolation fitting and Look-Up Table (LUT). Chen, et al were proposed method adopts smoothing spline approximation to precisely extract the specific system phase error from the reference phase according to the system's phase distribution properties [10]. But the spline interpolation fitting is multiple iterations, and time-consuming is expensive. LUT has been proved that the shape waviness is associated only with the nonlinearity of the Gamma curve of the DVP and CCD camera pair [7]. This intensity LUT can be constructed by either measuring the entire projected intensity levels or analyzing the fringe images statistically. Another LUT is presented by Zhang and Yau [11], and without pre-computing Gamma. The accuracy of phase compensation/depended on the length of the LUT because the measured phase was a real-number data set involving interpolation. The third method is Gamma curve correction. Guo et)al. employed statistical methods to analyze and correct for Gamma distortion by framing Gamma distortion as an uncertainty problem [12]. In their experiment high-frequency four-step PSP was adopted for a Gamma value of 1.4802, which was nôt a particularly chanlenging problem. Thang et al. were presented a pre-coding process is applied to correction the system for actual PSP system [13]. This method had advantages, such as simple and suited to conventional few-step phaseshifting algorithm. But it was using) a relatively large phase-shifting step (e.g., 20) to determine the correct phase distribution.

In this paper, we propose PSP mathematieal Gamma model for based on [2], but only using six Gamma-precoding uniform lumirance patterns for detecting the Gamma value involved in the DVP and CCD camera pair. Further the phase error compensation obtained by linear least square is using for reducing the phase shifting error after Gamma correction. In our experiments, the maximum phase error of PSP can be reduced by $75.0 \%$. After our Gamma calibration and phase error compensation processing, the maximum phase error is only $5.9 \%$ of without any correction or compensation.

\section{Principle of Gamma Pre-coding and Phase Distribution Least Square}

\subsection{Principle of Gamma Calibration}

In PSP as shown in Figure 1, a series of ideal fringe patterns, which gray-scales distribution of patterns as shown in Fig. 2, are generated by a computer and projected to the $3 \mathrm{D}$ object surface using a DVP. The CCD camera captures the reflected intensity from 3D object surface for further processing. Owing to the nonlinear gray-scale response of a PSP system including the overall influences of the DVP, object surface reflectivity, environment brightness, and the CCD camera, the captured fringe images will deviate from its ideal cosine waveform.

Typically, a pixel of the projected PSP cosine shifting pattern generated by a computer is expressed as

$$
\begin{aligned}
& I_{i}^{T}=I^{\prime}+I^{\prime \prime} \cos (\varphi+\delta(n)) \\
& \delta(n)=\frac{2 \pi}{N} i \quad i=1,2,3,4
\end{aligned}
$$


where, $I$ 'is the average of luminance in each step phase shifting patterns in this pixel, respectively and $I^{\prime \prime}$ is the changing value of phase shifting. $\varphi$ represents the fringe phase. $\delta(i)$ is the phase-shift amount. In formula (2), it acts as 4 fixed steps. And the fringe phase can then be described as

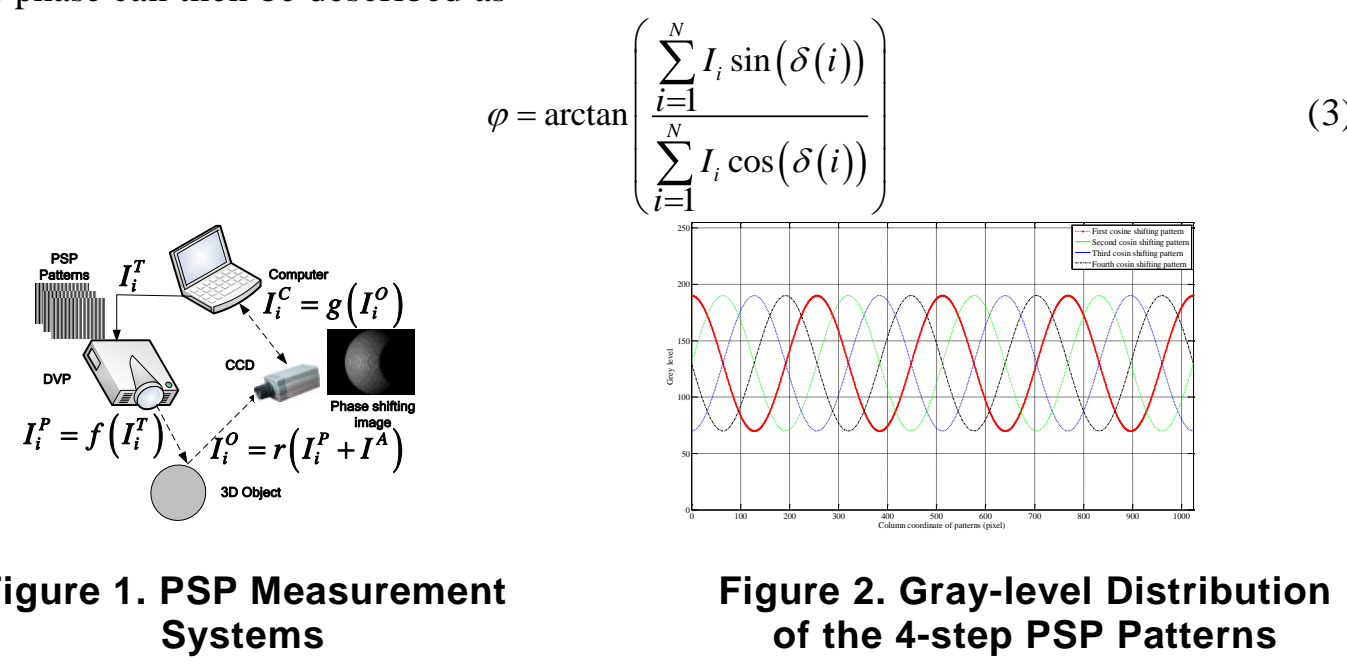

The patterns $I_{i}^{p}$ projected from DVP is distorted by the projector Gamma, and expressed as

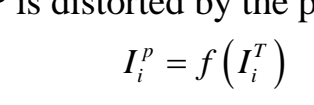

where $f()$ is the DVP response for input as $I_{i}^{t}$. The $I_{i}^{p}$ and ambient luminance $I^{A}$ is reflected by $3 \mathrm{D}$ object surface, and the reflected light $I_{i}^{O}$ modulated by $r$ the surface reflectance is expressed as

$$
I_{i}^{O}=r I_{i}^{P}+I^{A}
$$

and $I_{i}^{C}$ the gray-scale of distortion fringe image by CCD camera will be decoding in a computer, $I_{i}^{C}$ expresses as

$$
I_{i}^{C}=g\left(I_{i}^{o}\right)
$$

where $g()$ is the CED camera response function including the nonlinear distortion. As defining above, the nontinear relationship between the fringe image gray-scale with the fringe gray-level can simply expressed as follow

$$
\left\langle I_{i}^{C}\right\rangle=\left\langle I_{i}^{T}\right\rangle^{\gamma_{S}}
$$

where $\left.\left\langle I_{i}^{C}\right\rangle\right\rangle_{\text {is }}$ the normalized intensity value of the captured fringe image $I_{i}^{C}$, and $\left\langle I_{i}^{T}\right\rangle$ is the intensity normalized value governed by formula (1). $\gamma_{s}$ is the Gamma value of the entire system including DVP and CCD camera. This intensity transfer function is called Gamma distortion. It changes a fundamental frequency harmonic into multi-frequency. In order to facilitate analyzing Gamma distortion, we rewrite formula (1) as follows

$$
I_{i}^{T}=I^{\prime}\left[\left(1-\frac{I^{\prime \prime}}{I^{\prime}}\right)+\frac{I^{\prime \prime}}{I^{\prime}} \cos (\varphi+\delta(n))\right]
$$

According to the power model [14], the gamma distorted fringe image can be expressed as

$$
I_{i}^{C}=r I^{\prime}\left[\left(1-\frac{I^{\prime \prime}}{I^{\prime}}\right)+\frac{I^{\prime \prime}}{I^{\prime}} \cos (\varphi+\delta(n))\right]^{\gamma_{s}}+I^{A}
$$

The gamma distorted fringe $I_{i}^{C}$ can be transformed into a Fourier series 


$$
I_{i}^{C}=I_{0}+\sum_{j=1}^{n} \Psi_{j}^{o} \cos [j(\varphi-\delta(i))]
$$

where $I_{0}$ is the DC component of the $i$ th fringe image, ${ }_{j}{ }_{j}$ is the $j$ th harmonic coefficients. The binomial series theorem can express any the real power function of binomial as power series, i.e.

$$
(1+x)^{t}=\sum_{k=0}^{\infty}\left(\begin{array}{l}
t \\
k
\end{array}\right) x^{k}=\sum_{k=0}^{\infty} \frac{t(t-1)(t-2) \cdots(t-k+1)}{k !} x^{k}
$$

From formula (10), $q_{j}$ is expressed as follow

$$
q_{j}=r I^{\prime}\left(1-\frac{I^{\prime \prime}}{I^{\prime}}\right)^{\gamma_{s}} \sum_{m=0}^{\infty}\left(\begin{array}{c}
\gamma_{s} \\
2 m+j
\end{array}\right)\left(\frac{I^{\prime \prime}}{2\left(I^{\prime}-I^{\prime \prime}\right)}\right)^{2 m+j}\left(\begin{array}{c}
2 m+j \\
m
\end{array}\right)
$$

The harmonic coefficient $q_{j}^{\circ}$ has the following recurrence formula

$$
\left(\gamma_{s}+j+2\right) \tilde{I}_{j+2}=\left(\gamma_{s}-j\right) \tilde{I}_{j}+\frac{2\left({\underline{L^{\prime}}}^{\prime}-{\underline{I^{\prime \prime}}}^{\prime \prime}\right)}{\boldsymbol{Y}^{\prime \prime}}(j+1) \tilde{I}_{j+1}
$$

Based on the property of the harmonic coefficients in the Gamma distorted fringe in formula (12), the Gamma value can be determined if the coefficients $q_{j}$ are known. This is the principle of the Gamma calibration.

\subsection{Processing of Gamma Pre-coding}

According to formula (12), the practical fringe $M_{i}^{c}$ can be expressed in a Fourier series. Adopting DFT on it, the amplitude frequeney response can be obtained for a predefined fundamental frequency $f_{0}$

$$
I_{k}=\sum_{n=1}^{N-1} \exp \left(-i \frac{2 \pi}{N} n k\right) I_{i}^{C}
$$

According the theory of DFS the absolute value of the harmonic coefficients can be obtained

$$
80 \quad\left|I_{k}\right|=\frac{2}{N}\left|\tilde{I}_{j}\right|
$$

After determined he sign of harmonic coefficients, the Gamma value can obtain as follow

$$
\gamma_{s}=\frac{I^{\prime \prime}(j+2) \tilde{I}_{j+2}+j I^{\prime \prime} \tilde{I}_{j}-2\left(I^{\prime}-I^{\prime \prime}\right)(j+1) \tilde{I}_{j+1}}{I^{\prime \prime}\left(\tilde{I}_{j}-\tilde{I}_{j+2}\right)}
$$

Once $\mathbb{R}_{s} \mathrm{Is}$ obtained, setting it as an appropriate precoding value to the projected patterns. The same Gamma pre-coding detection is carried on all pixels of fringe. Apply Gamma pre-coding with $\gamma_{p}$ during fringe generation for all future measurements with the current setup.

\subsection{Processing of Linear Phase Expansion and PEC Obtained by Linear Least Square}

Combine with four-step phase-shifting method, linear phase unwrapping could be done as follows: Each set of fringe truncated after phase measuring can get

$$
\varphi_{w}(m, n, t)=\arctan \left[\frac{\Delta I_{42}(m, n, t)}{\Delta I_{13}(m, n, t)}\right]
$$


In the equation (16), $\Delta I_{42}=I_{4}(m, n)-I_{2}(m, n), \Delta I_{13}=I_{1}(m, n)-I_{3}(m, n),(m, n)$ is fringe image point row and column digital coordinate, and $t$ represents the unwrapped phase of $t$ th patterns.

The difference of truncate phase seen in equation (16)

$$
\Delta \varphi_{w}(m, n, t)=\arctan \left[\frac{\Delta I_{42}(t) \Delta I_{13}(t-1)-\Delta I_{13}(t) \Delta I_{42}(t-1)}{\Delta I_{13}(t) \Delta I_{13}(t-1)+\Delta I_{42}(t) \Delta I_{42}(t-1)}\right]
$$

The total expansion phase-difference of $s$ th image may be through the adjacent phase difference simple overlay to be

$$
\Delta \varphi_{u}(s, 0)=\sum_{t=1}^{s} \Delta \varphi_{w}(t, t-1)
$$

Actually, the equation (18) only use the first and sth image, sa measurement accuracy only depends on the error of $i=0$ and $t=s$ image. Phase error among them can offset. According to fringe projection profilometry, when we use 4 steps phase shifting and three different frequencies, we should take 12 images. But using the above theory, it can only use 8 images.

After Gamma calibration using the Gamma calibration, which is detail described in section 2.1 and 2.2, the phase-shifting error from the Gamma nonlinearity is suppressed to the minimum. But the light intensity is decayed with the optical path, and this decay will still induct the phase-shifting error Fortunately, this error sours is linear, because CCD camera sample is the mean intensity of interest area of 3D object surface. The linear least square could be reduced this phase-shiting error.

Defining $x$ is the measurement value of $f(y)$ and the linear relationship between $x$ and $f()$ can be expressed as

$$
f(x)=a+b x+c x^{2}
$$

Where $a, b$ and $c$ are the phase shifting error correction factors.

Assuming knowing $N$ points the phase-shifting error in $i$ th point pairs expressed as $\Delta_{i}=y_{i}-a-b \varphi(i)-\varphi(i)^{2}$. The sum of entire point pair residuals error is defined as $\Omega$

$$
\Omega=\sum_{j 0}^{N} \psi_{i}^{2}=\sum_{i=0}^{N-1}\left[y_{i}-\left(a+b \varphi(i)+c \varphi^{2}(i)\right)\right]^{2}=Y(a, b, c)
$$

According to the principle of least squares, the phase shifting error correction factor are obtained as follow formula

$$
\begin{aligned}
& {\left[\begin{array}{l}
a \\
b \\
c
\end{array}\right]=G^{-1} Y} \\
& G=\left(\begin{array}{ccc}
N & \sum_{i=0}^{N-1} \varphi(i) & \sum_{i=0}^{N-1} \varphi^{2}(i) \\
\sum_{i=0}^{N-1} \varphi(i) & \sum_{i=0}^{N-1} \varphi^{2}(i) & \sum_{i=0}^{N-1} \varphi^{3}(i) \\
\sum_{i=0}^{N-1} \varphi^{2}(i) & \sum_{i=0}^{N-1} \varphi^{3}(i) & \sum_{i=0}^{N-1} \varphi^{4}(i)
\end{array}\right) \quad Y=\left(\begin{array}{c}
\sum_{i=0}^{N-1} \varphi(i) \\
\sum_{i=0}^{N-1} \varphi(i) y(i) \\
\sum_{i=0}^{N-1} \varphi^{2}(i) y(i)
\end{array}\right)
\end{aligned}
$$$$
\text { Among them, }
$$

\section{3D Shape Measurement System and Experimental Results}

In order to verify the performance of the presented method in this paper, we programmed the 3D shape measurement experimental system of Figure 3. The system is 
composed of a Hitachi F22 camera with Computar M3Z1228C-MP camera lens and an Acer H7531D digital video projector. Camera images have a resolution of $1360 \times 1024$ pixels. The baseline between the camera and the projector is approximately $400 \mathrm{~mm}$. The angle between the optical axes of the camera and the projector is of about 30 degree.

Figure 4 shows the gray-scales response curve of this 3D shape measurement system. From this Figure, between 70 and 190, the gray-scale response is well, and the minimum and maximum gray-scales of the fringe patterns are selected respectively. The Gamma pre-coding initial is $\gamma_{p 1}=2.5$ and $\gamma_{p 2}=3.5 .3$ normalized intensity values $I_{i}^{T}$, which is the uniform luminance patterns generated by computer 1 , are $I_{1}^{T}=0.4, I_{2}^{T}=0.5$ and $I_{3}^{T}=0.6$. The CCD camera images as shown in from Figure 5 to Figure 10.

The Gamma pre-coding result is show in Figure 11. The brightness point is higher Gamma value and the darkness is lower Gamma value using in generation the 3D shape measurement fringe patterns by computer 1. After Gamma oalibration and ACDC linear compensation, the final phase error is shown as Figure 12 . This data is come from absolute coordinate map of a plane.

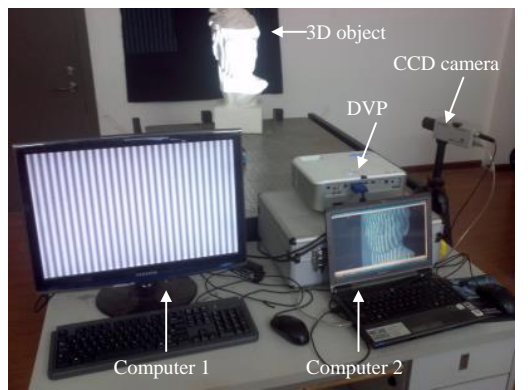

Figure 3. The Setup of 3D Shâpe Measurement System

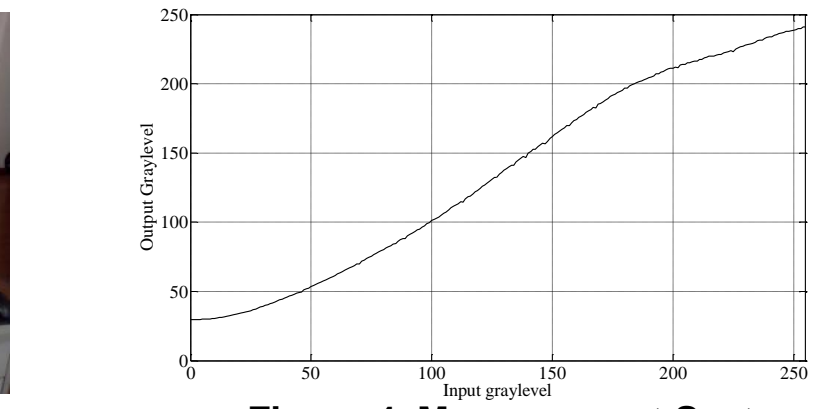

Figure 4. Measurement System Graylevel Response Function Curve

In Figure 12, the dot-line is the phase error without any correction or compensation, the maximum residuals is $0.235 \mathrm{rad}$. The dashed-line is the residuals error using precoding Gamma values shown as Figure 11, the maximum residuals is $0.176 \mathrm{rad}$, the precoding Gamma using 6 uniform luminance patterns can reduce the phase error to $75.0 \%$. And the solid line is the residuals error using the method proposed in this paper, the maximum phase error is $0.014 \mathrm{rad}$, the total residuals reduction is $94.1 \%$.

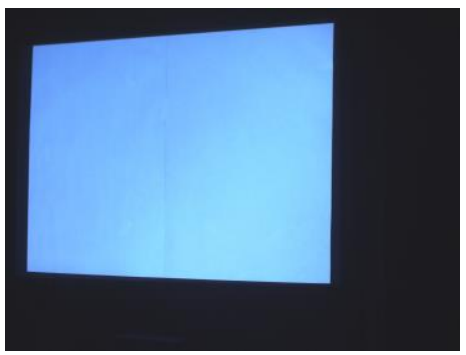

Figure 5 captured image

by CCD camera $\gamma_{p 1}=2.5$,

$I_{1}^{T}=0.4$

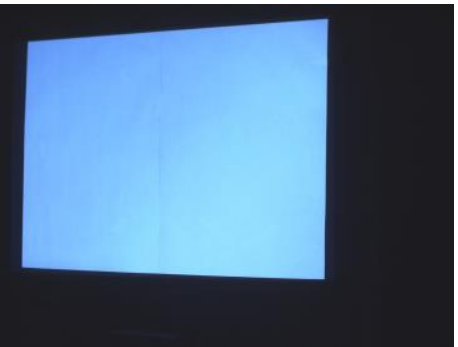

Figure 6 captured image by CCD camera $\gamma_{p 1}=2.5$, $I_{2}^{T}=0.5$

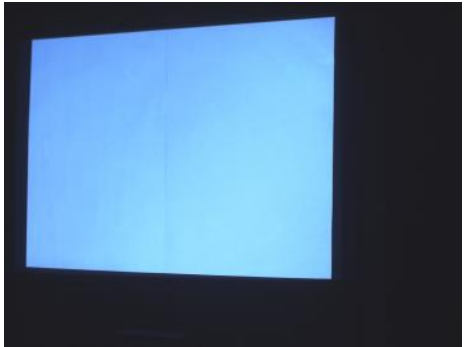

Figure 7 captured image by CCD camera $\gamma_{p 1}=2.5$, $I_{3}^{T}=0.6$ 

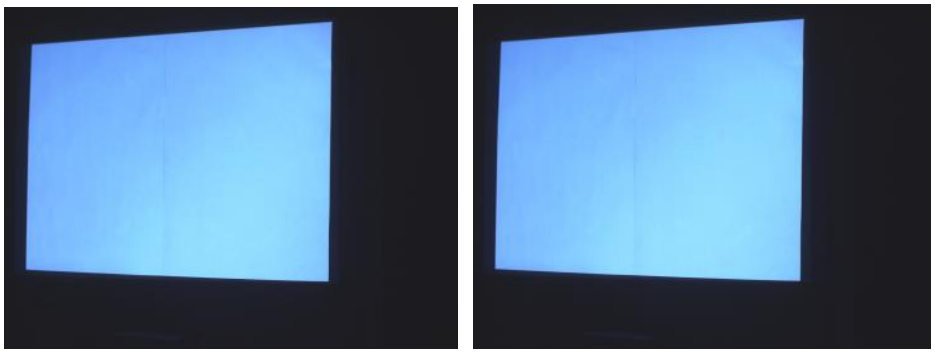

Figure 8. Captured Image Figure 9. Captured Image by CCD Camera $\gamma_{p 2}=3.5$, $I_{1}^{T}=0.4$ by CCD Camera $\gamma_{p 2}=3.5$, $I_{2}^{T}=0.5$

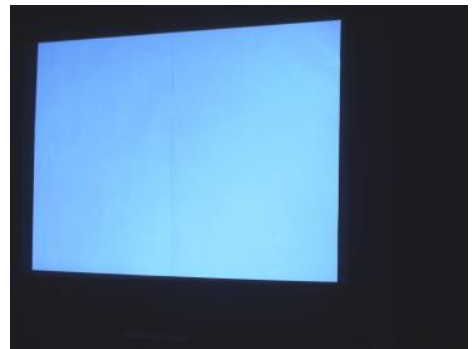

Figure 10. Captured Image by CCD

Camera $\gamma_{2}=3.5,1_{3}^{1}=0.6$

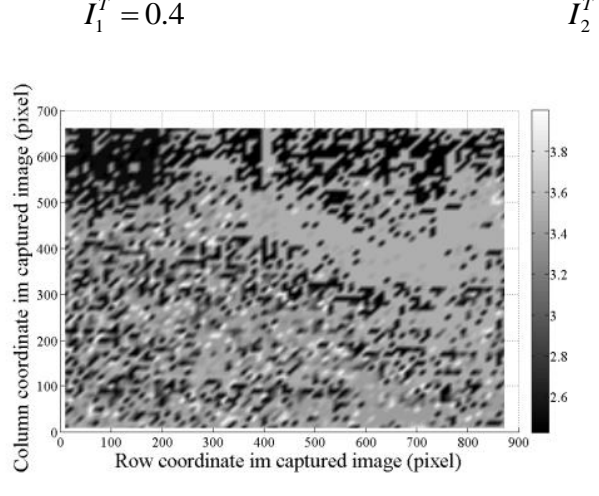

Figure 11. The Pre-coding Gamma .

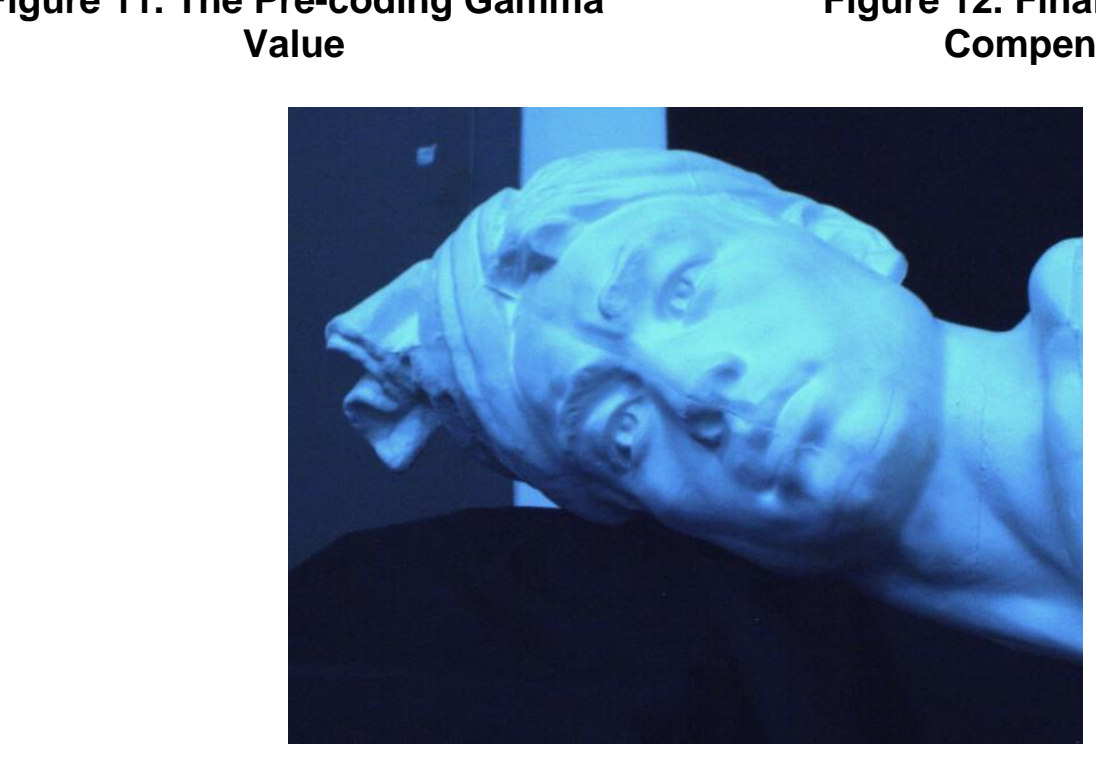

Figure 13. Tested Plaster Head

In order to the method proposed, a complex curve surface, a tested plaster head as shown in Figure 13, was reconstructed no using any correction or compensation shown as Figure 14 and Figure 15, respectively. From Figure 14, the reconstruction surface waviness is very clear, especially near the mouth of doll. From Figure 15 this waviness from the nonlinearity or linearity error source of the $3 \mathrm{D}$ shape measurement system is hardly any. 


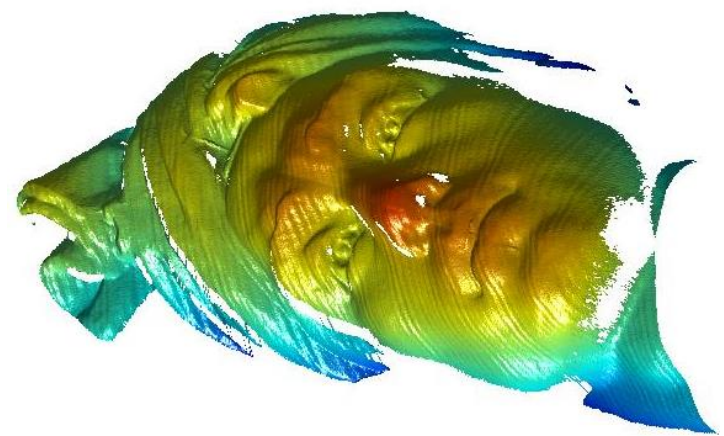

Figure 14. Reconstruction Surface without Any Compensation

\section{Conclusion}

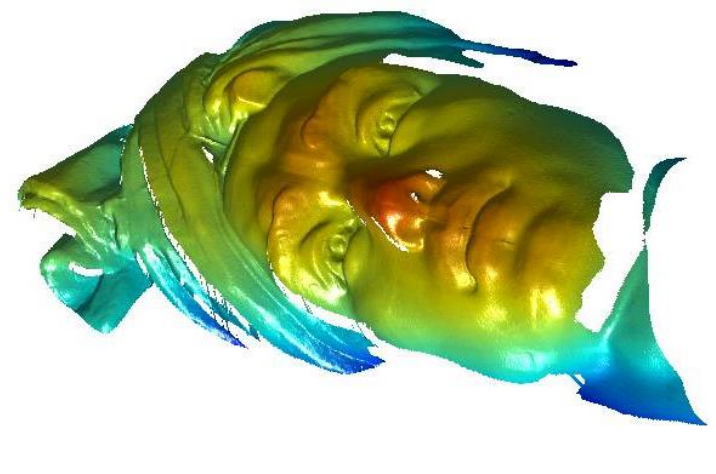

Figure 15. Reconstructed Surface using the Proposed Method in this S Paper

A novel phase error reduction combined Gamma calibration with linear least square is presented in this paper. The proposed method achieving the Gamma correction only using 6 uniform luminance patterns, the time-consuming in the same correction is fewer than other methods. The linear least square is further reducing the phase error. The total reduction of phase error is $94.1 \%$. The reconstruction surface of complex curve is hardly any the waviness, which is clearly display in the same reconstruction surface without any correction or compensation.

\section{Acknowledgements}

This research is supported by Technology Research Project of Heilongjiang Province Education Department (12521080) The constructive comments from the reviewers are of this paper gratefully acknowledged which have helped the author to improve the paper.

\section{References}

[1] Y. Xiao, Y. Cao, Y. Wu and S. Shi, “Opt. Eng.”, vol. 5, no. 52, (2013).

[2] X. Zhang, L. Zhu, Y. Li and D. Tu, "J. Opt. Soc. Am.”, A., vol. 6, no. 29, (2012).

[3] J. Salvi, S. Fernandez, T. Pribanic and X. Llado, "Pattern Recogn.", vol. 8, no. 43, (2010).

[4] Z. Li, Y. Shi C. Wang, D. Qin and K. Huang, "Opt. Commun.”, vol. 14, no. 282, (2009).

[5] L. C. CherrandX. L. Nguyen, "Meas. Sci. Technology", vol. 5, no. 21, (2010).

[6] C. Waddington and J. Kofman, "Opt. Laser. Eng.", vol. 2, no. 48, (2010).

[7] S.Zhang and P. S. Huang, "Opt. Eng.”, vol. 6, no. 46, (2007).

[8] Y Xu, L. Ekstrand, J. Dai and S. Zhang, “Appl. Opt.”, vol. 17, no. 50, (2011).

[9] M. Carocci, S. Lazzari, R. Rodella and G. Sansoni, "3D range optical sensor: analysis of the measurement errors and development of procedures for their compensation", Proc. SPIE 3313, Three-Dimensional Image Capture and Applications, (1998) January 24, San Jose, CA.

[10] X. Chen, J. Xi and Y. Jin, “Opt. Eng.”, vol. 11, no. 47, (2008).

[11] S. Zhang and S. Yau, “Appl. Opt.”, vol. 1, no. 46, (2007).

[12] H. Guo, H. He and M. Chen, “Appl. Opt.”, vol. 14, no. 43, (2004).

[13] T. Hoang, B. Pan, D. Nguyen and Z. Wang, "Opt. Lett.”, vol. 12, no. 35, (2010).

[14] K. Liu, Y. Wang, D. Lau, Q. Hao and L. Hassebrook, “J. Opt. Soc. Am.”, A., vol. 3, no. 27, (2010). 